\title{
BMJ Open High versus low energy administration in the early phase of acute pancreatitis (GOULASH trial): protocol of a multicentre randomised double-blind clinical trial
}

\begin{abstract}
Katalin Márta, ${ }_{1}^{1}$ Anikó N Szabó, ${ }^{1}$ Dániel Pécsi, ${ }^{1}$ Péter Varjú, ${ }^{1}$ Judit Bajor, ${ }^{1,2}$ Szilárd Gódi, ${ }^{1,2}$ Patrícia Sarlós, ${ }^{1,2}$ Alexandra Mikó, ${ }^{1,2}$ Kata Szemes, ${ }^{2}$ Mária Papp, ${ }^{3}$ Tamás Tornai, ${ }^{3}$ Áron Vincze, ${ }^{2}$ Zsolt Márton, ${ }^{2}$ Patrícia A Vincze, ${ }^{4}$ Erzsébet Lankó, ${ }^{4}$ Andrea Szentesi, ${ }^{1,5}$ Tímea Molnár, ${ }^{1}$ Roland Hágendorn, ${ }^{2}$ Nándor Faluhelyi, ${ }^{6}$ István Battyáni, ${ }^{6}$ Dezső Kelemen, ${ }^{7}$ Róbert Papp, ${ }^{7}$ Attila Miseta, ${ }^{8}$ Zsófia Verzár, ${ }^{9}$ Markus M Lerch, ${ }^{10}$ John P Neoptolemos, ${ }^{11}$ Miklós Sahin-Tóth, ${ }^{12}$ Ole H Petersen, ${ }^{13}$ Péter Hegyi, ${ }^{1,5}$ on behalf of the Hungarian Pancreatic Study Group
\end{abstract}

To cite: Márta K, Szabó AN, Pécsi D, et al. High versus low energy administration in the early phase of acute pancreatitis (GOULASH trial): protocol of a multicentre randomised doubleblind clinical trial. BMJ Open 2017;7:e015874. doi:10.1136/ bmjopen-2017-015874

- Prepublication history and additional material for this paper are available online. To view please visit the journal (http:// dx.doi.org/10.1136/bmjopen2017-015874).

Received 6 January 2017 Revised 21 July 2017 Accepted 25 July 2017

CrossMark

For numbered affiliations see end of article.

Correspondence to

Dr Péter Hegyi;

p.hegyi@tm-pte.org

\section{ABSTRACT}

Introduction Acute pancreatitis (AP) is an inflammatory disease with no specific treatment. Mitochondrial injury followed by ATP depletion in both acinar and ductal cells is a recently discovered early event in its pathogenesis. Importantly, preclinical research has shown that intracellular ATP delivery restores the physiological function of the cells and protects from cell injury, suggesting that restoration of energy levels in the pancreas is therapeutically beneficial. Despite several high quality experimental observations in this area, no randomised trials have been conducted to date to address the requirements for energy intake in the early phase of AP. Methods/design This is a randomised controlled twoarm double-blind multicentre trial. Patients with AP will be randomly assigned to groups A (30 kcal/ $/ \mathrm{kg} /$ day energy administration starting within 24 hours of hospital admission) or B (low energy administration during the first 72 hours of hospital admission). Energy will be delivered by nasoenteric tube feeding with additional intravenous glucose supplementation or total parenteral nutrition if necessary. A combination of multiorgan failure for more than 48 hours and mortality is defined as the primary endpoint, whereas several secondary endpoints such as length of hospitalisation or pain will be determined to elucidate more detailed differences between the groups. The general feasibility, safety and quality checks required for high quality evidence will be adhered to.

Ethics and dissemination The study has been approved by the relevant organisation, the Scientific and Research Ethics Committee of the Hungarian Medical Research Council (55961-2/2016/EKU). This study will provide evidence as to whether early high energy nutritional support is beneficial in the clinical management of AP. The results of this trial will be published in an open access way and disseminated among medical doctors.

Trial registration The trial has been registered at the ISRCTN (ISRTCN 63827758).
Strengths and limitations of this study

- Strength 1: This is a randomised controlled two-arm double-blind multicentre trial which provides the first type A evidence concerning the necessity of early energy intake for patients with AP.

- Strength 2: The study enjoys continuous support from an International Translational Advisory Board (ITAB) including several well established experts.

- Strength 3: Data will be separately handled by an Independent Data Management Board (IDMB).

- Strength 4: There are no unknown drugs/therapy used in the study, therefore no adverse and serious adverse events are expected.

- Limitation 1: In order to detect a treatment effect of at least $50 \%$ of the early treatment, a sample size of 957 subjects will be necessary to be recruited which will delay the final conclusion of the study.

- Limitation 2: The double-blind arrangement of the study requires many staff members working on the project which may limit the number of joining centres.

\section{BACKGROUND}

Acute pancreatitis (AP) is an inflammatory disease of the exocrine pancreas which is life threatening in its severe form. Unfortunately, while the overall mortality of AP is around $2-5 \%$, and in its severe form $25-57 \%$, no specific treatment is available. Besides the limited interest of pharmacological companies, the main reasons are (1) the small number of research teams in the field and (2) the lack of collaboration between basic and clinical scientists. Importantly, many new 
therapeutic targets were identified in the last decade with clear translational merits. ${ }^{1-8}$ One of the main highlights among them is the discovery of energy depletion in the early phase of AP. ${ }^{13-5}$ 7-17

It has been shown that, almost independently of the aetiological factors, the early phase of AP is almost the same. Bile acids, ethanol, fatty acids and the latter's metabolite fatty acid ethyl esters cause mitochondrial damage and ATP depletion in pancreatic ductal and acinar cells, driving the cells to death and causing pancreatic necrosis. ${ }^{1}{ }^{1} 41^{10-14}$ 18-31 Very importantly, restoration of ATP levels in both cell types prevented cell death and at least partially restored their function. ${ }^{19}$ In experimental pancreatitis models the same observations have been revealed. ${ }^{10-21}$ Although these experimental observations clearly suggest that restoration of the energy level could be a therapeutic tool in AP, this has not been translated into clinical trials.

One of the best and most physiological way of delivering energy to a patient is enteral nutrition (EN). Not surprisingly, besides fluid resuscitation this is almost the only way to significantly reduce mortality in $\mathrm{AP}^{22-33}$ Recent analyses of prospectively collected data from 600 patients with AP showed that the mortality is $27 \%$ with $\mathrm{EN}$ and $57 \%$ without $\mathrm{EN}$ in the severe form (SAP). ${ }^{34}$ Importantly, EN decreases mortality but also reduces the frequency of multiorgan failure and the need for interventions in patients with $\mathrm{SAP}^{35}$ No data are available on whether early or on-demand nutrition/energy supply is beneficial in SAP. The recently published Dutch PYTHON study suggests that there is no difference between early and on-demand enteral tube feeding in SAP, but patients may have received an insufficient amount of energy at the early phase of the disease. ${ }^{36}{ }^{37}$ In the early EN group, patients received over $20 \mathrm{kcal} / \mathrm{kg} /$ day only from day 3 onwards whereas, in the on-demand group, they received energy supplementation only from day $6 .{ }^{37}$ In mild and moderate AP (MAP) much less information is available concerning the usefulness of EN. There is a large variety of protocols on EN in MAP. Immediate oral feeding, ${ }^{38}$ nasojejunal feeding ${ }^{39-41}$ and nasogastric feeding ${ }^{42}{ }^{43}$ have all been used. Notably, immediate oral feeding significantly decreased the length of hospital stay. ${ }^{38}$ Early (within 24hours) nasogastric EN was well tolerated and reduced the intensity and duration of abdominal pain, decreased the necessity for opiates and almost completely eliminated the risk of oral food intolerance. ${ }^{42}$ In order to obtain stronger evidence of the usefulness of early EN in MAP and SAP, we performed a systematic review and meta-analysis which showed that early EN can be beneficial in both MAP and SAP. ${ }^{35}$ However, we also realised the lack of multicentre randomised control trials addressing energy intake in the early phase of AP.

The main objective of this trial is to determine whether early energy supplementation is beneficial to patients with AP. Our hypothesis is that early energy supplementation will prevent the cells from death or decrease the size of necrosis if it occurs. This will decrease the systemic immune response that will result in a lower frequency of multiorgan failure and mortality. To prove this concept, a randomised clinical trial involving all patients with AP is needed.

\section{METHODS \\ Design}

This is a randomised controlled two-arm double-blind multicentre trial. Patients with AP will be randomly assigned to groups A (high energy administration starting within 24 hours of hospital admission) and B (no energy administration after 24 hours of hospital admission).

\section{Trial organisation, committees and boards}

GOULASH is designed and coordinated by the Centre for Translational Medicine at the University of Pécs and the Hungarian Pancreatic Study Group (HPSG). HPSG was established in 2011 in order to stimulate research in pancreatic diseases. To date, HPSG has published the relevant guidelines of pancreatic diseases in order to improve patient care in the field of pancreatology ${ }^{44-47}$ and has initiated four prospective clinical trials (EASY, PREPAST, APPLE and PINEAPPLE).$^{48-51}$

The following committees and boards will be involved:

\section{Steering committee (SC)}

The Steering committee (SC) will be led by PH (gastroenterologist, internal medicine specialist). The members will be KM (medical doctor, full time employee on the project), ÁV (gastroenterologist, internal medicine specialist), ZM (intensive care specialist), TM (clinical research specialist), AS (multidisciplinary unit specialist), MP (gastroenterologist, internal medicine specialist), NF (radiologist), DK (surgeon) and IB (interventional radiologist). SC will make decisions concerning all relevant questions including the dropouts during the study.

\section{International translational advisory board (ITAB)}

The committee will include a gastroenterologist (MML), a surgeon (JPN) and basic scientists (MST, OHP). ITAB will continuously monitor the progress of the study and will give advice to the SC.

The study was designed by the SC and ITAB. The study is financially sponsored by the University of Pécs, the Hungarian Academy of Sciences and the National Research, Development and Innovation Office. Neither sponsors were involved in the design of the study, and they will have no access to the database management or to the randomisation code.

\section{Study population}

All patients diagnosed with AP will be informed of the possibility of taking part in the GOULASH study. After the consent form is signed, a computer using a block randomisation protocol will randomise the patients (figure 1). 


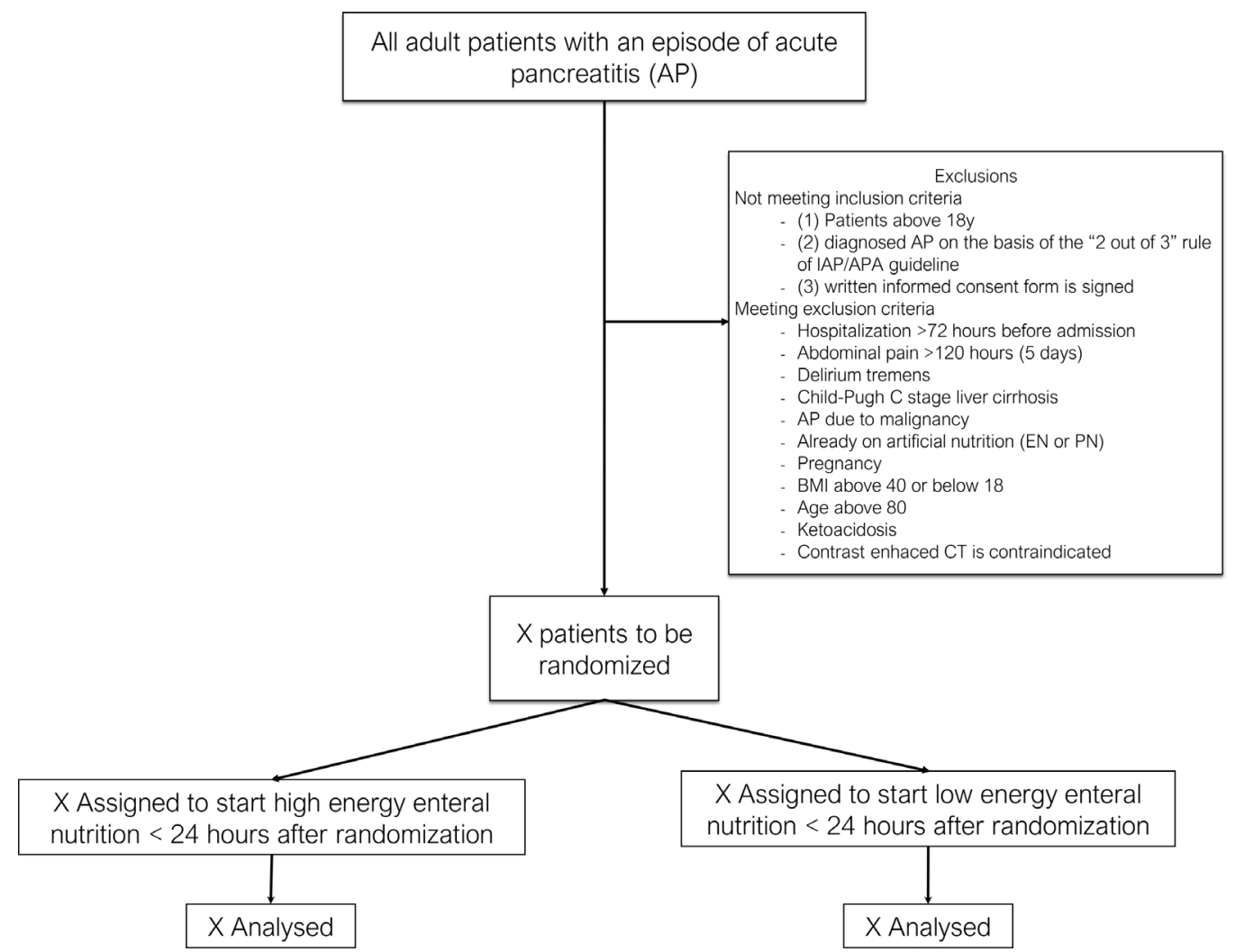

Figure 1 Flow chart of participants according to the SPIRIT 2013 statement. $^{53}$

\section{Inclusion criteria}

The inclusion criteria are: (1) patients over 18 years of age; (2) diagnosed AP on the base of the ' 2 out of 3' criteria of the IAP/APA guideline $^{52}$ : (a) upper abdominal pain; (b) serum amylase or lipase $>3 \mathrm{x}$ upper limit of normal range; (c) characteristic findings on pancreatic imaging; however those patients without abdominal pain will be excluded because the onset of AP cannot be determined; (3) signed written informed consent form.

\section{Exclusion criteria}

The exclusion criteria are: (1) hospitalisation 72 hours before admission; (2) abdominal pain >120 hours (5 days); (3) delirium tremens; (4) Child-Pugh C stage liver cirrhosis; (5) AP due to malignancy; (6) already on artificial nutrition (EN or PN); (7) pregnancy; (8) BMI $>40$ or $<18$; (9) age >80 years; (10) ketoacidosis; and (11) whenever CT with contrast is contraindicated.

\section{Sample size}

Sample size calculation was based on the Hungarian National Registry operated by the HPSG. Our recent analyses indicated that multiorgan failure existing for more than 48 hours arises in 9\%, whereas mortality occurs in $2.8 \%$ of all patients with $\mathrm{AP} .{ }^{34}$ Altogether they represent around $10 \%$ of all AP patients. In order to detect a treatment effect of at least $50 \%$ of the early treatment, a sample size of 957 subjects will be necessary to be recruited using a $10 \%$ drop-out rate, $80 \%$ power and $95 \%$ significance level. The calculation was performed by the independent data management and biostatistics provider company (IDMB, Adware Research Ltd, Balatonfüred, Hungary).

\section{Randomisation}

In each centre participants will be divided into two groups receiving one of the two study treatments. The allocation of participants to the different groups will be carried out based on predefined randomisation lists created separately for each recruiting centre. The randomisation lists will be prepared with a block size of 4 and with an allocation ratio of 1:1.

\section{Duration}

The planned starting date of the study is 1 January 2017 and the planned finishing date of the study is 1 January 2020 .

\section{Blinding}

The medical staff (eg, those taking the measurements such as blood pressure, examining health records for events such as abdominal pain, reviewing and interpreting examination results such as X-ray or CT) and the patient receiving the intervention will be blinded to knowledge of treatment assignment. The person providing the intervention cannot be blinded in this study. Sealed envelopes ensure the allocation sequence. Nutritional support equipment will be covered until the fourth day to ensure that only the person who made the randomisation will know into which group the patient was enrolled. 


\begin{tabular}{|r|c|c|c|c|c|c|c|c|}
\hline & \multicolumn{7}{|c|}{ STUDY PERIOD } & \begin{tabular}{c} 
Close-out \\
\hline
\end{tabular} \\
\hline
\end{tabular}

Figure 2 Schedule of enrolment, interventions and assessments according to the SPIRIT 2013 statement. ${ }^{53}$ Patients will be randomised to group A (high energy) or B (low energy). Online supplementary figure 1 Form A contains the parameters collected on admission. Online supplementary figure 2 Form B contains parameters collected every day during hospitalisation. Online supplementary figure 3 Form $\mathrm{C}$ contains parameters collected 1 month after hospital discharge.

\section{Intervention}

Based on the currently available guidelines, enteral feeding can be started at any time for patients with AP. In addition, no calorie restriction/order has been described. Therefore, both groups can be regarded as being treated within accepted practice recommendations.

In this study, early high energy administration will be the intervention. Patients will be randomised to group A or B (see figure 2).

\section{Groups}

In group A, high energy will be delivered after admission. Patients will receive a $10 \mathrm{Ch}$ nasogastric $(\mathrm{NG})$ or nasojejunal (NJ) feeding tube on admission. EN will be immediately started as follows: on day 0 (from admission until the start of EN, which can vary from 2 to 24 hours): calorie intake will be $0 \mathrm{kcal} / \mathrm{kg} /$ day; from day 1 , high energy enteral tube feed $30 \mathrm{kcal} / \mathrm{kg}$ /day will be provided until the oral feeding starts. In group B, low energy administration will be delivered after hospital admission. Patients will receive a NG or $\mathrm{NJ}$ feeding tube at admission as described above. On day 0 (from admission until the start of $\mathrm{EN}$ ): calorie intake will be $0 \mathrm{kcal} / \mathrm{kg} /$ day; on day $1,0 \mathrm{kcal} / \mathrm{kg} /$ day; on day 2,10 $\mathrm{kcal} / \mathrm{kg} /$ day, on day $3,20 \mathrm{kcal} / \mathrm{kg} /$ day and from day 4,30 $\mathrm{kcal} / \mathrm{kg} /$ day will be delivered until the oral feeding starts.
However, between groups A and B only the amount of calories administered will be different. Patients will receive the same amount of fluid and ions during EN (see below).

Ingredients of enteral tube feed: high energy enteral tube feed $(100 \mathrm{~mL})$

Energy

$150 \mathrm{kcal}(630 \mathrm{~kJ})$, protein $6 \mathrm{~g}(16 \% \mathrm{E})$, carbohydrate $18.3 \mathrm{~g}$ $(49 \% \mathrm{E})$, fat $5.8 \mathrm{~g}(35 \% \mathrm{E})+$ minerals: $134 \mathrm{mg}$ sodium, $201 \mathrm{mg}$ potassium, $108 \mathrm{mg}$ calcium, $108 \mathrm{mg}$ phosphorus, $34 \mathrm{mg}$ magnesium, $100 \mathrm{mg}$ chloride $(0 \% \mathrm{E})$. In this study we will use Nutrison Energy (Numil Ltd, Budapest, Hungary), which is a registered product in Hungary (reg. number: 1217).

\section{Zero energy enteral tube feed $(100 \mathrm{~mL})$}

Energy

$0 \mathrm{kcal}(0 \mathrm{~kJ})$, protein $0 \mathrm{~g}$, carbohydrate: $0 \mathrm{~g}$, fat $0 \mathrm{~g}+$ minerals: $134 \mathrm{mg}$ sodium, $201 \mathrm{mg}$ potassium, $108 \mathrm{mg}$ calcium, $108 \mathrm{mg}$ phosphorus, $34 \mathrm{mg}$ magnesium, $5.562 \mathrm{~g}$ chloride $(0 \% \mathrm{E})$. In this study the local institutional pharmacy will provide it in accordance with the Hungarian regulations. Whenever 10 or $20 \mathrm{kcal} / \mathrm{kg} /$ day calories are to be delivered, a mixture of the abovementioned two solutions will be used. 


\section{Type of enteral tube}

Patients neither vomiting nor having gastric fluid retention $>250 \mathrm{~mL}$ will receive a NG tube. Patients either vomiting or having gastric fluid retention $>250 \mathrm{~mL}$ will receive a NJ tube (placement will be done either endoscopically or radiologically). In case of Glasgow Coma Score (GCS) 14 or lower in a patient who is not intubated, the NG tube will be replaced by a NJ tube (risk of aspiration). Abdominal X-ray will be used to check the position of the tube.

Start of mixed feeding (around $2620 \mathrm{kcal}$ ): $1000 \mathrm{~mL}$ tap water distributed for 24 hours and $300 \mathrm{~g}$ (around 1900 kcal) biscuits/toasts/low fat meal (containing at least $75 \%$ carbohydrate) orally plus enteral tube feed $(480 \mathrm{~mL}$, $720 \mathrm{kcal} /$ day) will be started on the day when: (1) abdominal pain has ceased for at least 6 hours before the new day started; (2) the C-reactive protein (CRP) level has started decreasing; and (3) the amylase or lipase level has started decreasing.

Start of total feeding (around $2000 \mathrm{kcal}$ ): if the patient has no symptoms during the mixed oral/enteral feeding and the CRP, amylase or lipase levels are not rising again, total feeding (according to local policy) can be started.

\section{Other issues}

The speed of EN will be different for patients depending on the body weight, however, the maximum speed of EN cannot exceed $65 \mathrm{~mL} /$ hour. In case of difficulties reaching an intake of $30 \mathrm{kcal} / \mathrm{kg} /$ day calories (if the patient's body weight is $>75 \mathrm{~kg}$ ), additional intravenous calories will be added using Sterofundin G. A maximum of $2000 \mathrm{~mL}$ ( $400 \mathrm{kcal}$ ) can be delivered in this way. If NG feeding is not tolerated, the NG tube will be replaced by a $\mathrm{NJ}$ tube as described above. If $\mathrm{NJ}$ feeding is not tolerated, EN will be reduced by $50 \%$ and increased again gradually until tolerated. If the re-increasing process is still not tolerated, total parenteral nutrition (TPN) will be started to reach the required energy target. In patients with SAP, TPN must be delivered via a central venous catheter.

\section{Other treatment of subjects}

General treatment indicated by the IAP/APA guideline will be utilised. ${ }^{52}$

\section{Discharge of patients}

Uniformisation of the length of hospital stay is necessary to avoid bias concerning length of hospital stay. Readmission within 1 week after discharge has to be considered as the same hospital admission. Patients will be counted as discharged from hospital/from the study when (1) oral feeding is tolerated for 24 hours; (2) amylase/lipase levels are not elevated after total enteral feeding; (3) CRP level is $<50 \mathrm{mg} / \mathrm{L}$; (4) abdominal pain has completely resolved; and (5) no other pancreatitis-related complication requiring hospitalisation is detected.

\section{Endpoints}

The following primary endpoints will be calculated: a combination of multiorgan failure for more than
48 hours and mortality. The following secondary endpoints will be analysed: (1) pancreatic necrosis; (2) nutrition-related complications (eg, diarrhoea, aspiration pneumonia, pneumothorax due to central TPN catheter placement); (3) need for conversion from NG to $\mathrm{NJ}$ feeding tube; (4) need for conversion from $\mathrm{EN}$ to TPN; (5) days until the start of total feeding; (6) use of antibiotics; (7) pain relapse; (8) CRP; (9) white blood cells; (10) procalcitonin; (11) infection; (12) length of hospital stay; (13) need for ICU admission; (14) length of ICU therapy; (15) organ failure; (16) complications; (17) costs calculation. Notably, only direct costs will be calculated that include all medications, services, salaries of healthcare professionals, equipment and day care costs.

\section{Monitored parameters during hospitalisation}

There will be a large variety of parameters monitored during the study (eg, medical history, physical examination, laboratory tests, diagnostic imaging, therapy, interventions). Form A will contain the parameters collected on admission (online supplementary figure 1). Form B will contain parameters collected every day during hospitalisation (online supplementary figure 2). Form C will contain parameters collected 1 month after hospital discharge (online supplementary figure 3). For details see supplementary materials or web page (http://www. pancreas.hu/en/studies/goulash), which will be available from February 2017. Data collection on the case report form (CRF) will be done electronically (see data management).

\section{Data management and statistical analyses \\ Data handling}

Data will be handled by the IDMB. Electronic CRF (eCRF) will be used. The Investigator will ensure that the data in the eCRF are accurate, complete and legible. Detailed data flow will be described in a Data Management Plan (DMP). Data from completed eCRFs will be validated under the direction of the Data Manager at IDMB according to a Data Cleaning Plan (DCP). Any missing, implausible or inconsistent recordings in the eCRFs will be referred back to the Investigator using a data query form (DQF), and be documented for each individual subject before clean file status is declared. All changes to eCRFs will be recorded. Before Data Base Lock the Data Review Meeting will decide and document necessary steps related to any issue in the database and define the analysis sets. Members of the Data Review Meeting are a delegated investigator, biostatistician and data manager. Adverse events will be coded using MedDRA (AdWare Research Ltd), who will act as IDMB, works according to GCP, GLP, FDA 21CFR PART11 and other relevant regulatory requirements. AdWare Ltd.has GLP and ISO 9001 certificates.

\section{Study populations}

Three analysis populations will be defined: 
Safety Analysis Set (SAS): all patients enrolled in the study.

Per Protocol Set (PPS): all enrolled patients who finished the study conforming to the requirements of the study protocol.

Intention to Treat (ITT): all randomised participants who start on a treatment, excluding consent withdrawals.

\section{Withdrawal of a subject from PPS}

Any participants/investigators and the IDMB can submit recommendations for dropouts from the PPS group with reasons given to the SC. All recommendations will be filed. The SC will discuss all the information and, if the alteration in the protocol would be expected to have any bearing on the interventions and outcomes of the study, the patient will not be included in the final per-protocol analysis. Automatic dropout from the per-protocol group shall be ordered if: (1) any of the exclusion criteria are diagnosed during the course of AP; (2) at least 50\% of the energy requirement is not achieved on any days during the study; (3) parameters required for answering the primary endpoints are missing; or (4) serious medical reasons not related to pancreatitis occur (eg, accidents, stroke).

\section{Applied software}

Statistical analysis will be performed using SAS 9.2 or SPSS 19 (or later) statistical packages; Microsoft MS Word will be used for reporting.

\section{Statistical methods}

Baseline patient and disease characteristics will be analysed using descriptive analysis. Demographic and baseline characteristics will be summarised for the overall study population. Continuous variables will be described by mean, median, SD and ranges and categorical variables will be described by absolute and relative frequencies. A graphical presentation of efficacy variables will be prepared, if applicable. Descriptive statistics for both the primary and secondary parameters will be analysed similarly. Mean changes (with 95\% CI) from baseline to end-of-study visit will also be presented. $\chi^{2}$ tests will be applied to compare proportions between the different groups. Mortality/extended multiorgan failure will be investigated using the Kaplan-Meier analysis method, while subgroup comparisons will be performed using the $\chi^{2}$ or Fisher's exact test, as appropriate. For safety data, descriptive statistics and individual listings of adverse events will also be presented.

\section{Subgroups}

The following subgroups will be made during statistical analyses: (1) ages ( $<40$ years, $40-59$ years, $60-80$ years); (2) BMI (<20, 20-24, 25-29, 30-35, >35); (3) start of abdominal pain before admission ( $\leq 24$ hours, 24-48 hours, $\geq 48$ hours); (4) severity of the disease SAP and MAP. In all subgroup analyses, aetiologies will be done descriptively. No confirmatory statistical testing will be applied. Hence, statistical tests and $p$ values attached to them will be regarded as descriptive and not as tests of hypotheses.

Details of the applied statistical tests will be described in the Statistical Analysis Plan.

\section{Early quality assessment}

Early quality assessment check will be performed on the first 100 patients. The IDMB (AdWare Ltd) will perform an independent assessment of the trial-related documents and activities, with the aim of ensuring the respect of subjects' rights, safety and well-being and to guarantee the plausibility of the clinical data. The similarity of the groups at baseline will also be checked. The IDMB will report to the SC. The SC will discuss all the information and, if the differences would be expected to have any bearing on the interventions and outcomes of the study or the overall dropout rate from PPS is $>20 \%$ of all participants who were randomised or allocated into each group or the differential dropout rate is $>15 \%$ between the arms, the study needs to be reassessed and the IDMB will make recommendations regarding either re-evaluation of power calculation, extension of recruitment period, extension of number of study centres or termination of trial.

\section{Interim analyses and premature termination of the study}

The IDMB can also recommend to stop the trial early for ethical reasons if one of the groups clearly shows evidence of a significant benefit. An interim analysis will be performed on the primary endpoint when 50\% of patients have been randomised and discharged from hospital. The interim analysis will be performed by the IDMB, who will report to the SC.

The Haybittle-Peto boundary approach will be used. If the interim analysis shows a probability of $\leq 0.001$ that a difference as extreme between the treatments is found, given that the null hypothesis is true, then the trial will be stopped early.

\section{Centres}

The trial will start in two centres (University of Debrecen and University of Pécs), after which the study is open for other centres. In all cases the IDMB will make an audit of the centre and will report to the SC. The SC has the right to decide whether the centre meets the required quality to join the study. Compulsory requirements for a centre are: (1) it needs to treat at least 50 patients with AP a year; (2) it needs to have all the equipment required for the study; (3) besides the regular medical team, the centre has to appoint at least one doctor and one nurse/administrator fully available for the trial with no additional commitments which can interfere with her/his duty when her/his availability is required; (4) the blinding described above can be fully utilised; (5) all persons need to attend a preliminary meeting where all the details concerning the studies are discussed fully and have qualified as investigators in a GCP course. Centres wishing to join need 
to send a letter of intent to the corresponding author by email.

\section{Publication policy}

Centres providing more than 25 patients can provide two authors to the authorship list. Every additional 25 patients will give the opportunity to nominate an additional author.

\section{Feasibility}

As a general protocol for the treatment of AP at the Centre for Translational Medicine at the University of Pécs, patients with AP receive early EN (using a NG tube). Patients receive $50 \mathrm{~mL}$ Nutrison Energy per hour starting immediately when they arrive to the ward from the Emergency Department. Patient data between the period 1 January 2016 to 31 May 2016 were analysed and the following observations were noted. (1) In $85 \%$ of all AP admissions early EN could have been started within 24 hours; in $15 \%$ of cases it was not achievable due to delayed transfer to the ward or vomiting. In these cases, patients received a NG tube later or they received a NJ tube whenever X-ray assistance was available. (2) Around $80 \%$ of NG-fed patients tolerated NG feeding without any complications. For the rest of the patients who had gastric retention or vomiting, NG feeding was stopped and they received a NJ tube whenever X-ray assistance was available.

(3) Comparing the outcome (rate of severity, mortality, necrosis, intervention, etc) of this treatment protocol with the nil per os protocol used in most Hungarian hospitals showed that patients enjoyed benefits with no risk of early enteral feeding, which data confirm the literature described in the introduction. About 250 patients at the University of Pécs and about 150 patients at the University of Debrecen are admitted annually. Therefore, if no other institution joins the study, it can be completed within 3 years.

\section{Safety}

Since no unknown drugs/therapy are used in the study, no adverse or serious adverse events are expected/interpretable that would be attributable to the intervention during the trial. In this trial the IDMB will examine safety variables after every 16 patients have completed. Moreover, investigators will report adverse or serious adverse events on a separate form which has to be sent to the IDMB and SC. The SC will discuss and, if the adverse effect is confirmed, it will be reported to the relevant institutional and national ethical committee (http:// www.ett.hu/tukeb.htm).

\section{Additional information and future plan}

Blood samples (serum and plasma) from all patients will be stored in order to study laboratory parameters later if required (eg, the laboratory could not measure it), and in order to build up a biobank for later clinical studies to which all participants will be given informed consent. The samples will be stored at $-80^{\circ} \mathrm{C}$. A follow-up study (called GOULASH PLUS) is under preparation in order to follow the patients for up to 5 years after the study. The study protocol will also be published.

\section{DISCUSSION}

Here we report the protocol of a prospective double-blind randomised controlled trial to study the effects of early energy restoration in AP. The preclinical studies ${ }^{19}$ and meta-analyses suggest that early energy supplementation should be beneficial. Our main hypothesis is that elevating the energy level of acinar and ductal cells will prevent these cells from injury, therefore decreasing the extent of necrosis during AP. Since both the local and systemic complications (immune response) largely depend on the extent of the necrosis, we propose that this intervention will reduce multiorgan failure and mortality in AP as well. Although nutritional interventions for patients with mild pancreatitis are probably not needed, we must involve all patients with AP in the study. It has to be highlighted that the main aim of the study is not to find new treatments for MAP or SAP but to prevent the development of SAP. This is the reason why severity cannot be a selection criterion but has to be the primary endpoint. Concerning ethical issues, this study has very low risk for patients. The enteral solution (Nutrison Energy) used in this study is widely used in several diseases related to malnutrition in patients and has almost no contraindications, therefore no adverse events are expected during the trial.

\section{ETHICS AND DISSEMINATION}

The trial is registered at the ISRCTN registry (ISRCTN63827758) and received relevant ethical approval with the reference number 55961-2/2016/EKU issued by the Scientific and Research Ethics Committee of the Medical Research Council. At the end of the project we will disseminate our results to the medical community and will publish our results via open access.

\section{CONCLUSION}

This study provides the first type A evidence concerning the necessity of energy intake for patients with AP. This protocol is the first version of the trial completed on 24 May 2017.

\section{Author affiliations}

${ }^{1}$ Institute for Translational Medicine, University of Pécs, Pecs, Hungary

${ }^{2} 1$ st Department of Internal Medicine, University of Pécs, Pécs, Hungary

${ }^{3}$ 2nd Department of Internal Medicine, University of Debrecen, Debrecen, Hungary

${ }^{4}$ Department of Pharmaceutics and Central Clinical Pharmacy, University of Pécs,

Pécs, Hungary

${ }^{5}$ MTA-SZTE Translational Gastroenterology Research Group, Szeged, Hungary

${ }^{6}$ Department of Radiology, University of Pécs, Pécs, Hungary

${ }^{7}$ Surgery Clinic, University of Pécs, Pécs, Hungary

${ }^{8}$ Department of Laboratory Medicine, University of Pécs, Pécs, Hungary

${ }^{9}$ Department of Emergency Medicine, University of Pécs, Pécs, Hungary

${ }^{10}$ Department of Medicine A, University Medicine Greifswald, Greifswald, Germany

${ }^{11}$ Department of Molecular and Clinical Cancer Medicine, University of Liverpool,

Liverpool, UK 
${ }^{12}$ Center for Exocrine Disorders, Department of Molecular and Cell Biology, Boston University Henry M. Goldman School of Dental Medicine, Boston, Massachusetts, USA

${ }^{13}$ Medical Research Council Group, Cardiff School of Biosciences, Cardiff University, Cardiff, UK

Contributors All authors were involved in the study design, edited the manuscript, read and approved the final manuscript. During the study KM, ANS, DP and PV are going to randomise the patients and ensure the blinding. AM, KS, JB, SG, MP, PS, ZV and TT are going to manage the treatment of the patients. ÁV will be responsible for the organisation, quality and timing of the endoscopic treatments, ZM and $\mathrm{RH}$ for the intensive care, NF and IB for the imaging and interventional radiology, DK and RP for the surgical treatment if needed. PAV and EL will prepare the nutritional solutions. MML, JPN, MST and OHP are members of ITAB. TM and AS will be members of SC. PH and KM drafted the manuscript.

Funding Centre costs (IT, biostatistics, trial organization, etc) are covered by the University of Pécs, Momentum Grant of the Hungarian Academy of Sciences (LP2014-10/2014); and Economic Development and Innovation Operative Programme Grant and Highly Cited Publication Grant of the National Research, Development and Innovation Office (GINOP-2.3.2-15-2016-00015, KH-125678). Since no additional treatment is necessary for the study, the general healthcare costs are covered by the National Healthcare System.

Competing interests None declared.

Patient consent Obtained.

Ethics approval The Scientific and Research Ethics Committee of the Medical Research Council, 55961-2/2016/EKU.

Provenance and peer review Not commissioned; externally peer reviewed.

Open Access This is an Open Access article distributed in accordance with the Creative Commons Attribution Non Commercial (CC BY-NC 4.0) license, which permits others to distribute, remix, adapt, build upon this work non-commercially, and license their derivative works on different terms, provided the original work is properly cited and the use is non-commercial. See: http://creativecommons.org/ licenses/by-nc/4.0/

(C) Article author(s) (or their employer(s) unless otherwise stated in the text of the article) 2017. All rights reserved. No commercial use is permitted unless otherwise expressly granted.

\section{REFERENCES}

1. Criddle DN, Murphy J, Fistetto G, et al. Fatty acid ethyl esters cause pancreatic calcium toxicity via inositol trisphosphate receptors and loss of ATP synthesis. Gastroenterology 2006;130:781-93.

2. Petersen $\mathrm{OH}$, Tepikin AV, Gerasimenko JV, et al. Fatty acids, alcohol and fatty acid ethyl esters: toxic $\mathrm{Ca} 2+$ signal generation and pancreatitis. Cell Calcium 2009;45:634-42.

3. Hegyi P, Petersen OH. The exocrine pancreas: the acinar-ductal tango in physiology and pathophysiology. Rev Physiol Biochem Pharmacol 2013;165:1-30.

4. Mukherjee R, Mareninova OA, Odinokova IV, et al. Mechanism of mitochondrial permeability transition pore induction and damage in the pancreas: inhibition prevents acute pancreatitis by protecting production of ATP. Gut 2016;65:1333-46.

5. Hegyi P, Wilschanski M, Muallem S, et al. CFTR: A new horizon in the pathomechanism and treatment of pancreatitis. Rev Physiol Biochem Pharmacol 2016;170:37-66.

6. Hegyi P. Blockade of calcium entry provides a therapeutic window in acute pancreatitis. J Physiol 2016;594:257.

7. Maléth J, Hegyi P. Ca2+ toxicity and mitochondrial damage in acute pancreatitis: translational overview. Philos Trans $R$ Soc Lond B Biol Sci 2016;371:20150425.

8. Szentesi $A$, Tóth $E$, Bálint $E$, et al. Analysis of research activity in gastroenterology: pancreatitis is in real danger. PLOS One 2016;11:e0165244.

9. Judák L, Hegyi P, Rakonczay Z, et al. Ethanol and its non-oxidative metabolites profoundly inhibit CFTR function in pancreatic epithelial cells which is prevented by ATP supplementation. Pflugers Arch 2014;466:549-62.

10. Gukovskaya AS, Pandol SJ, Gukovsky I. New insights into the pathways initiating and driving pancreatitis. Curr Opin Gastroenterol 2016:429-35.
11. Chakraborty M, Hickey AJ, Petrov MS, et al. Mitochondrial dysfunction in peripheral blood mononuclear cells in early experimental and clinical acute pancreatitis. Pancreatology 2016:16:739-47.

12. Maléth J, Hegyi P, Rakonczay Z, et al. Breakdown of bioenergetics evoked by mitochondrial damage in acute pancreatitis: mechanisms and consequences. Pancreatology 2015;15(4 Suppl):S18-S22.

13. Huang W, Cash N, Wen L, et al. Effects of the mitochondria-targeted antioxidant mitoquinone in murine acute pancreatitis. Mediators Inflamm 2015;2015:1-13.

14. Criddle DN. The role of fat and alcohol in acute pancreatitis: a dangerous liaison. Pancreatology 2015;15(4 Suppl):S6-S12.

15. Trumbeckaite S, Kuliaviene I, Deduchovas O, et al. Experimental acute pancreatitis induces mitochondrial dysfunction in rat pancreas, kidney and lungs but not in liver. Pancreatology 2013;13:216-24.

16. Mittal A, Hickey AJ, Chai CC, et al. Early organ-specific mitochondrial dysfunction of jejunum and lung found in rats with experimental acute pancreatitis. HPB 2011;13:332-41.

17. Sung KF, Odinokova IV, Mareninova OA, et al. Prosurvival $\mathrm{Bcl}-2$ proteins stabilize pancreatic mitochondria and protect against necrosis in experimental pancreatitis. Exp Cell Res 2009;315:1975-89.

18. Odinokova IV, Sung KF, Mareninova OA, et al. Mitochondrial mechanisms of death responses in pancreatitis. $J$ Gastroenterol Hepatol 2008;23(Suppl 1):S25-S30.

19. Gukovskaya AS, Gukovsky I, Jung Y, et al. Cholecystokinin induces caspase activation and mitochondrial dysfunction in pancreatic acinar cells. Roles in cell injury processes of pancreatitis. J Biol Chem 2002;277:22595-604.

20. Kui B, Balla Z, Végh ET, et al. Recent advances in the investigation of pancreatic inflammation induced by large doses of basic amino acids in rodents. Lab Invest 2014;94:138-49.

21. Biczó G, Hegyi P, Dósa $\mathrm{S}$, et al. The crucial role of early mitochondrial injury in L-lysine-induced acute pancreatitis. Antioxid Redox Signal 2011;15:2669-81.

22. Guillou PJ. Enteral versus parenteral nutrition in acute pancreatitis. Baillieres Best Pract Res Clin Gastroenterol 1999;13:345-55.

23. Eatock FC, Brombacher GD, Steven A, et al. Nasogastric feeding in severe acute pancreatitis may be practical and safe. Int $J$ Pancreatol 2000;28:23-30.

24. Abou-Assi S, O'Keefe SJ. Nutrition in acute pancreatitis. $J$ Clin Gastroenterol 2001;32:203-9.

25. Shi D, Zhang CW, Jiang JS, et al. Enteral nutrition in treatment of severe acute pancreatitis. Hepatobiliary Pancreat Dis Int 2002;1:146-9.

26. Yousaf M, McCallion K, Diamond T. Management of severe acute pancreatitis. Br J Surg 2003;90:407-20.

27. Eckerwall G, Olin H, Andersson B, et al. Fluid resuscitation and nutritional support during severe acute pancreatitis in the past: what have we learned and how can we do better? Clin Nutr 2006;25:497-504.

28. McClave SA, Chang WK, Dhaliwal R, et al. Nutrition support in acute pancreatitis: a systematic review of the literature. JPEN J Parenter Enteral Nutr 2006;30:143-56.

29. Pupelis G, Snippe K, Plaudis $\mathrm{H}$, et al. Early oral feeding in acute pancreatitis: an alternative approach to tube feeding. Preliminary report. Acta Chir Belg 2006;106:181-6.

30. Besselink MG, van Santvoort HC, Witteman BJ, et al. Management of severe acute pancreatitis: it's all about timing. Curr Opin Crit Care 2007;13:200-6.

31. DiMagno MJ, DiMagno EP. New advances in acute pancreatitis. Curr Opin Gastroenterol 2007:6:592-9.

32. Hegazi RA, O'Keefe SJ. Nutritional immunomodulation of acute pancreatitis. Curr Gastroenterol Rep 2007;9:99-106.

33. Jiang $\mathrm{K}$, Chen $\mathrm{XZ}$, Xia Q, et al. Early nasogastric enteral nutrition for severe acute pancreatitis: a systematic review. World J Gastroenterol 2007;13:5253-60.

34. Párniczky A, Kui B, Szentesi A, et al. Prospective, Muticentre, Nationwide clinical data from 600 cases of acute pancreatitis. PLOS One 2016:11:e0165309.

35. Márta K, Farkas N, Szabó I, et al. Meta-analysis of early nutrition: the benefits of enteral feeding compared to a nil per os diet not only in severe, but also in mild and moderate acute pancreatitis. Int $\mathrm{J} \mathrm{Mol}$ Sci 2016;17:1691.

36. Bakker OJ, van Santvoort HC, van Brunschot S, et al. Pancreatitis, very early compared with normal start of enteral feeding (PYTHON trial): design and rationale of a randomised controlled multicenter trial. Trials 2011;12:73.

37. Bakker OJ, van Brunschot S, van Santvoort HC, et al. Early versus on-demand nasoenteric tube feeding in acute pancreatitis. $N$ Engl $J$ Med 2014;371:1983-93. 
38. Eckerwall GE, Tingstedt BB, Bergenzaun PE, et al. Immediate oral feeding in patients with mild acute pancreatitis is safe and may accelerate recovery: a randomized clinical study. Clin Nutr 2007;26:758-63.

39. Abou-Assi S, Craig K, O'Keefe SJ. Hypocaloric jejunal feeding is better than total parenteral nutrition in acute pancreatitis: results of a randomized comparative study. Am J Gastroenterol 2002;97:2255-62.

40. Oláh A, Belágyi $T$, Issekutz $A$, et al. Randomized clinical trial of specific lactobacillus and fibre supplement to early enteral nutrition in patients with acute pancreatitis. Br J Surg 2002;89:1103-7.

41. McClave SA, Greene LM, Snider HL, et al. Comparison of the safety of early enteral vs parenteral nutrition in mild acute pancreatitis. JPEN J Parenter Enteral Nutr 1997;21:14-20.

42. Petrov MS, Mcllroy K, Grayson L, et al. Early nasogastric tube feeding versus nil per os in mild to moderate acute pancreatitis: a randomized controlled trial. Clin Nutr 2013;32:697-703.

43. Ma J, Pendharkar SA, O'Grady G, et al. Effect of nasogastric tube feeding vs nil per os on dysmotility in acute pancreatitis: results of a randomized controlled trial. Nutr Clin Pract 2016;31:99-104.

44. Dubravcsik Z, Farkas G, Hegyi P, et al. [Autoimmune pancreatitis. Evidence-based management guidelines of the Hungarian Pancreatic Study Group]. Orv Hetil 2015;156:292-307.

45. Hritz I, Czakó L, Dubravcsik Z, et al. [Acute pancreatitis. Evidencebased practice guidelines, prepared by the Hungarian Pancreatic Study Group]. Orv Hetil 2015;156:244-61.
46. Párniczky A, Czakó L, Dubravcsik Z, et al. [Pediatric pancreatitis. Evidence-based management guidelines of the Hungarian Pancreatic Study Group]. Orv Hetil 2015;156:308-25.

47. Takács T, Czakó L, Dubravcsik Z, et al. [Chronic pancreatitis. Evidence-based management guidelines of the Hungarian Pancreatic Study Group]. Orv Hetil 2015;156:262-88.

48. Dubravcsik Z, Madácsy L, Gyökeres T, et al. Preventive pancreatic stents in the management of acute biliary pancreatitis (PREPAST trial): pre-study protocol for a multicenter, prospective, randomized, interventional, controlled trial. Pancreatology 2015;15:115-23.

49. Hritz I, Hegyi P, Severity EA. Early Achievable Severity (EASY) index for simple and accurate expedite risk stratification in acute pancreatitis. J Gastrointestin Liver Dis 2015;24:177-82.

50. Párniczky A, Mosztbacher D, Zsoldos F, et al. Analysis of pediatric pancreatitis (APPLE trial): pre-study protocol of a multinational prospective clinical trial. Digestion 2016;93:105-10.

51. Zsoldos F, Párniczky A, Mosztbacher D, et al. Pain in the early phase of pediatric pancreatitis (PINEAPPLE Trial): pre-study protocol of a multinational prospective clinical trial. Digestion 2016;93:121-6.

52. Working Group IAP/APA Acute Pancreatitis Guidelines. IAP/APA evidence-based guidelines for the management of acute pancreatitis. Pancreatology 2013;13(4 Suppl 2):e1-15.

53. Chan AW, Tetzlaff JM, Altman DG, et al. SPIRIT 2013 statement: defining standard protocol items for clinical trials. Ann Intern Med 2013;158:200-7. 